\title{
Çift Ters Sarkaç Sisteminin Kontrolü için PID ve LQR Kontrolcü Tasarımlarının Modellenmesi
}

\author{
Süleyman Çeven $^{1}$, Ahmet Albayrak ${ }^{2 *}$ \\ ${ }^{1}$ Düzce Üniversitesi, Düzce Meslek Yüksekokulu, Elektronik ve Otomasyon Teknolojisi Bölümü, Düzce, Türkiye (ORCID: 0000-0002-8970-4826) \\ ${ }^{2}$ Düzce Üniversitesi, Teknoloji Fakültesi, Bilgisayar Mühendisliği Bölümü, Düzce, Türkiye (ORCID: 0000-0002-2166-1102)
}

(Bu yayın 26-27 Haziran 2020 tarihinde HORA-2020 kongresinde sözlü olarak sunulmuştur.)

(DOI: 10.31590/ejosat.780070)

ATIF/REFERENCE: Çeven, S., \& Albayrak, A. (2020). Çift Ters Sarkaç Sisteminin Kontrolü için PID ve LQR Kontrolcü Tasarımlarının Modellenmesi. Avrupa Bilim ve Teknoloji Dergisi, (Special Issue), 323-330.

$\ddot{O} \mathbf{z}$

Günümüzde, ters sarkaç sisteminde denge sağlama özellikle kontrol sistemleri üzerine çalışma yapan araştırmacıların kontrol teori ve yöntemlerini kıyasladıkları güncel bir konudur. Ters sarkaç sistemi kontrol edilebilirlik açısından kararsız ve doğrusal olmayan sistemler arasında yer almaktadır. Yapısının karmaşıklığı ve kontrollerinin zorluğu nedeniyle birçok ileri düzey kontrol teorisi bu sistemler üzerinde uygulanarak kontrolcülerin performansları üzerinde değerlendirme yapılabilmektedir. Bu çalışmada, PID ve LQR kontrolcü yöntemleri MATLAB ortamında matematiksel olarak modellenen bir çift eklemli ters sarkaç üzerinde uygulanmış ve kontrolcü performansları karşılaş̧ırılımıştır. PID ve LQR kontrol yöntemlerinin uygulanabilirliği üzerinde elde edilen sonuçlar değerlendirilmiştir.

Anahtar Kelimeler: Çift eklemli ters sarkaç, PID kontrolcü, LQR kontrolcü, kontrol sistemleri.

\section{Modelling of PID and LQR Controller for Stability and Position Control in Double Inverted Pendulum System}

\begin{abstract}
Nowadays, stability of the inverted pendulum system is an up-to-date topic in which researchers working on control systems compare control theories and methods. The inverse pendulum systems are unstable and nonlinear systems in terms of controllability. Due to the complexity of the structure and the difficulty of the control process, many advanced control theories can be applied on these systems to improving the performance of the controllers. In this study, PID and LQR controller methods were applied on a double inverted pendulum modeled in MATLAB environment and their controller performances were compared. The results via experimental studies were evaluated on the applicability of PID and LQR control methods.
\end{abstract}

Keywords: Double inverted pendulum, PID controller, LQR controller, Control systems.

\section{Giriş}

Günümüzde otomasyon ve robotik alanlarındaki ilerlemeler ile birlikte insan faktörünün en aza indirgendiği otonom sistemler, kullanım açısından artış göstermektedir. Otonom sistemlerin artmasıyla birlikte kontrol teorisi üzerinde çalışmaların artarak devam etmesi gerekliliği öne çıkmaktadır. Özellikle zor ve karmaşık hareketlerin analiz edilmesi amaçlanan robotik uygulamalar, doğrusal olmayan, kararsız sistemlerde güçlü kontrol sistemlerine ihtiyaç duyulmaktadır. Bu çalışmada, modellenen Çift Eklemli Ters Sarkaç (ÇETS) üzerinde sarkacın denge kontrolü farklı yöntemlerle sağlanmış ve yöntemlerin performansları ortaya konulmuştur. Ters sarkaç sistemi, karmaşık mekanik yapısı, kararsız ve doğrusal olmayan bir sistem olması sebebiyle otomatik kontrol alanında yapılan çalışmalarda kullanılmaktadır (Önen et al., 2019). Literatürde yeni bir kontrolcü sistemi veya var olan sistemlerin geliştirilmesi gibi çalışmalarda önerilen kontrol sistemi performansının karşılaştırılması ters sarkaç sistemlerinde denenerek yöntemin performansı

* Sorumlu Yazar: Düzce Üniversitesi, Teknoloji Fakültesi, Bilgisayar Mühendisliği Bölümü, Düzce, Türkiye, ORCID: 0000-0002-2166-1102, ahmetalbayrak@duzce.edu.tr 
kolaylıkla değerlendirilebilmektedir (Sheng et al., 2008). Konuya ilişkin yapılan bir çalışmada LQG (Linear-Quadratic-Gaussian) kontrol yöntemi bir ÇETS modeli üzerinde uygulanmıştır (Zheng et al., 2016). LQR (Linear-Quadratic-Regulator) kontrolcüsüne ek olarak parametrelerin değerlendirilmesi işleminde Kalman filtreleme metodu kullanılarak LQG kontrolcü elde edilmektedir. ÇETS sistemi uzay durum matrisleri kullanılarak modellenmiş ve uygulamaların tamamı MATLAB/Simulink üzerinde gerçekleştirilmiştir. Çift eklemli ters sarkacın dengelenmesinde kullanılan etkili yöntemlerden olan LQR kontrolcüde optimizasyon yaklaşımları ile kontrol probleminin çözümü sağlanmaktadır (Önen et al., 2019). Parçacık Sürüsü Optimizasyonu (PSO), Yapay Arı Kolonisi Algoritması (YAK) ve Genetik Algoritma (GA) yöntemleri ile LQR kontrolcüsünün $Q$ ve R matrisleri güncellenmekte ve kazanç ifadesi olan $\mathrm{K}$ matrisi elde edilmektedir. Çalışmada farklı yöntemler sistem üzerinde uygulanarak sistem cevaplarının karşılaştırılması yapılmıştır. Genel olarak ters sarkacın matematiksel modeli üzerinde yapılan çalışmaların yanı sıra MATLAB/SimMechanics araç kutusu kullanılarak ters sarkaç sistemi modellenebilmekte ve kontrol yöntemleri uygulanabilmektedir (Tinkir et al., 2010).

Literatürde ters sarkaç mekanik olarak Matlab üzerinde modellenmiş ve model çıkarımlarının tamamı Lagrange eşitlikleri kullanılarak oluşturulmuştur. Ters sarkacın dengede kalması için Fuzzy Type-2 yöntemi modellenen sisteme uygulanmış ve PID yöntemi ile karşılaş̧ırılmıştır. Sonuç olarak Fuzzy Type-2 yöntemi daha başarılı olduğu yazarlar tarafindan ortaya konulmuştur. ÇETS'in dinamik hareket modeli üzerinden modellendiği başka bir çalışmada füzyon fonksiyonu ve bulanık mantık denetleyici kullanılarak sistemin denge kontrolünün yapılması amaçlanmıştır (Zhang et al., 2012). Dinamik matematiksel eşitlikler sonucu elde edilen uzay durum matrisleri üzerinde tasarlanan kontrolcü yapısı uygulanmış ve başarı sağlanmıştır. Tasarlanan bulanık mantık denetleyicisi giriş parametreleri referans değişkenlerdeki hata ve hatadaki değişim olarak tanımlanmıştır. Ters sarkaç sistemlerinde en uygun kontrolcü yapısının farklı yöntemler uygulanarak değerlendirildiği bir yüksek lisans tezinde ters sarkaç sisteminde LQG yönteminin LQR yönteminden daha başarılı sonuç verdiği aktarılmaktadır (Priyadarshi, 2013). Yapılan bir diğer çalışmada uzay durum geri beslemeli kutup yerleşim kontrol yöntemi kullanılmış ve gerçekleştirilen bir ÇETS sisteminde uygulanmıştır (Wu et al., 2008). Sarkaç sisteminde bulunan arabanın hareketi bir elektrik motoru kullanılarak sağlanmıştır. Sarkacın eklemlerine ait açısal parametreler kullanılan açı ölçer donanımlar tarafından sağlanmaktadır. Ters sarkaç sistemlerini denge kontrollerinde ayrıca melez bulanık mantık ve yapay sinir ağından (ANFIS) kontrol yöntemleri kullanılmaktadır (Sheng et al., 2008). Yapılan çalışmalarda LQR yöntemi ile melez çalışan bir ANFIS modeli yöntem olarak öne sürülmüş ve sistem çıkışları izlenerek performansı değerlendirilmiştir. Ters sarkaç sistemlerinde mekanik sürtünme kontrolcünün başarısının arttırılması için dikkate alınması gereken bir parametredir (Nejadfard et al., 2013). Ters sarkacın mekanik sistemlerindeki sürtünmeyi ihmal etmek kontrolcü tasarımında eksiklik oluşturacağından özellikle model tabanlı kontrol yaklaşımları kontrolcü performansını düşürecektir. Yapılan çalışmada bir ÇETS sistemi Lagrange eşitlikleri kullanılarak matematiksel olarak modellenmiş, sürtünme giderici yeni bir kontrol yöntemi önerilmiştir. Önerilen yöntemde sezgisel bir bulanık yapay sinir ağı modeli geliştirilmiştir. Yapay sinir ağı eğitimi LOLIMOT (Local Linear Model Trees) öğrenme algoritması ile sağlanmıştır. Sinir ağının eğitilmesi için 2000 veriden oluşan bir veri seti kullanılmıştır. Başka bir çalışmada ise LQR metodu ile bir çift eklemli ters sarkacın denge kontrolü yapılmış ve bu işlemden elde edilen veriler ile bir yapay sinir ağı modeli eğitilmiş ve kontrol bu ağ modeli üzerinden gerçekleştirilmiştir (Arbo et al., 2014). Bulanık mantık temelli RNA genetik algoritma yöntemi ile bir çift eklemli ters sarkacın denge kontrolü başka bir çalı̧̧mada sağlanmış ve yöntem çıktıları başka yöntemlerle karşılaştırılarak yeni bir kontrolcü önerisi yapılmıştır. Önerilen yöntemin diğer güncel yöntemlerden daha iyi sonuç verdiği sistem tepki sonuçlarından anlaşılmaktadır (Sun et al., 2015). Genel olarak bulanık mantık kontrolcünün kullanıldığı başka bir çalışmada ise bulanık mantık üyelik fonksiyonlarının sınırları genetik algoritma ile belirlenmiş ve sistem performansı değerlendirilmiştir. Bulanık mantığa ek olarak genetik algoritma kullanımının performansı artırdığı öne sürülmektedir (Ding et al., 2009).

Bu çalışmada, Matlab ortamında modellenen bir ÇETS sistemi üzerinde PID ve LQR kontrolcüleri ile kontrol sağlanmış, ÇETS sisteminin başlangıç konumdan "0" konumuna ulaşması sürecinde kullanılan farklı kontrol yöntemlerine ait başarı performansları izlenmiştir. ÇETS'ın LQR kontrolcü ile kontrol edilmesi için sistem uzay-durum modeli, PID kontrolcü ile kontrol edilmesi için ise Matlab/Simulink üzerinde ters sarkacın modellemesi yapılmışıır. Her iki kontrolcü performans sonuçları Simulink üzerinde modellenen sistemde yapılan simülasyonlar ile elde edilmektedir. Bu çalışmanın literatüre katkısı PID ve LQR yöntem performanslarının ÇETS üzerinde değerlendirilmesi ve karşılaştırılmasıdır. Makalenin ikinci bölümünde ÇETS matematiksel modeli ve eşitlikler, üçüncü bölümünde PID ve LQR kontrolcü tasarımları, dördüncü bölümünde deneysel çalışmalar ve beşinci bölümünde sonuçlar paylaşılmaktadır.

\section{2. Çift Eklemli Ters Sarkacın Matematiksel Modeli}

Bu çalışmada modellenen ÇETS modeli Şekil 1'de, sistem modellenmesinde ihtiyaç duyulan parametreler ise Tablo 1'de verilmektedir. Şekil 1'de verilen sistem bir araba mekanizmasına bağlı alt ve üst sarkaçtan oluşmaktadır. Sistemi oluşturan arabanın ağırlığı $\mathrm{m}_{1}$, alt sarkaç ağırlığı $\mathrm{m}_{2}$ ve üst sarkaç ağırlığı ise $\mathrm{m}_{3}$ olarak tanımlanmıştır. Sistemde verilen $\mathrm{L}_{1}$ ve $\mathrm{L}_{2}$ parametreleri sırasıyla alt sarkaç boyu ve üst sarkaç boyunu temsil etmektedir. $\theta_{1}$ ve $\theta_{2}$ ise sırasıyla alt sarkaç ve üst sarkacın y ekseni ile yaptığı açısal büyüklüktür. ÇETS hareketini sadece $\mathrm{x}$ ekseninde yapabildiği düşünüldüğünde, sisteme uygulanan $\mathrm{F}$ kuvvetinin yönünün de $\mathrm{x}$ ekseni üzerinde olmas gerekmektedir. Sisteme harici olarak etki eden tek fiziksel parametre F kuvveti olacaktır. Sistemin üç serbestlik derecesine sahip olması ve bu üç ayrı sistem değişkenini yalnızca F kuvvetinin etkilemesi göz önünde bulundurulduğunda ÇETS denge kontrolü probleminin çözülmesinde kontrol parametresi F kuvveti, referans parametreler ise sarkacın konumu, alt sarkaç açısı ve üst sarkaç açısı olmalıdır. Çalışma Matlab platformunda simülasyon şeklinde gerçekleştirildiği için, modelde kullanılan bazı parametreler literatürden alınmıştır. 


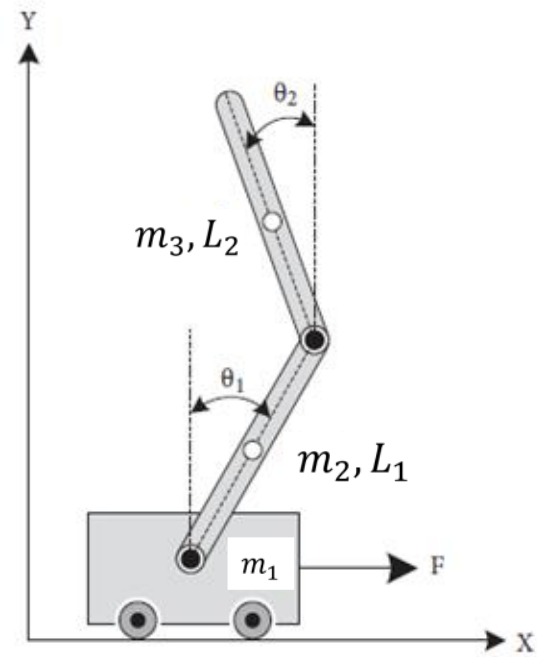

Şekil 1. ÇETS modeli (Önen et al., 2019)

Tablo 1. Sistem parametreleri (Sheng et al., 2008)

\begin{tabular}{|c|c|c|c|}
\hline Parametre & Simge & Birim & Büyüklük \\
\hline Araba ăgırlı̆̆g & $\mathrm{m}_{1}$ & $\mathrm{~kg}$ & 1.32 \\
\hline Alt sarkaç ăğrllğgl & $\mathrm{m}_{2}$ & $\mathrm{~kg}$ & 0.132 \\
\hline Üst sarkaç ağırllı̆g & $\mathrm{m}_{3}$ & $\mathrm{~kg}$ & 0.208 \\
\hline Sistem etki kuvveti & $\mathrm{F}$ & $\mathrm{N}$ & - \\
\hline Yerçekimi ivmesi & $\mathrm{g}$ & $\mathrm{m} / \mathrm{s}^{2}$ & 9.81 \\
\hline Alt sarkaç açısı & $\theta_{1}$ & $\mathrm{rad}$ & - \\
\hline Üst sarkaç açısı & $\theta_{2}$ & $\mathrm{rad}$ & - \\
\hline Alt sarkaç boyu & $\mathrm{L}_{1}$ & $\mathrm{~m}$ & 0.09 \\
\hline Üst sarkaç boyu & $\mathrm{L}_{2}$ & $\mathrm{~m}$ & 0.27 \\
\hline
\end{tabular}

Sistemin dinamik hesaplamaları Eşitlik 1'de verilmektedir.

$$
\begin{aligned}
& a \ddot{x}+\cos \theta_{1} \ddot{\theta}_{1}+c \cos \theta_{2} \ddot{\theta}_{2}-b \sin \theta_{1} \dot{\theta}_{1}{ }^{2}-c \sin \theta_{2} \dot{\theta}_{2}{ }^{2}=F \\
& b \cos \theta_{1} \ddot{x}+d \delta \ddot{\theta}_{1}+e \cos \left(\theta_{2}-\theta_{1}\right) \ddot{\theta}_{2}+e \sin \left(\theta_{1}-\theta_{2}\right) \ddot{\theta}_{2}{ }^{2}-b g \sin \theta_{1}=0 \\
& c \cos \theta_{2} \ddot{x}+e \cos \left(\theta_{2}-\theta_{1}\right) \ddot{\theta}_{1}+f \ddot{\theta}_{2}+e \ddot{\theta}_{1}{ }^{2} \sin \left(\theta_{2}-\theta_{1}\right)-c g \sin \theta_{2}=0
\end{aligned}
$$

Eşitlik 1'de verilen $a=\left(m_{1}+m_{2}+m_{3}\right), b=\left(\frac{1}{2} m_{1} L_{1}+m_{2} L_{1}\right), c=\left(\frac{1}{2} m_{2} L_{2}\right), d=\left(\frac{1}{4} m_{1} L_{1}{ }^{2}+m_{2} L_{1}^{2}\right), e=\left(\frac{1}{2} m_{2} L_{1} L_{2}\right), f=$ $\left(m_{2} L_{2}{ }^{2}\right)$ eşitliklerini temsil etmektedir. Sistemin dinamik eşitliklerinden faydalanarak sistem durum uzay modeli oluşturulabilmektedir. Sisteme ait durum-uzay modeli Eşitlik 2'de verilmektedir.

$$
\begin{aligned}
& \dot{x}=A x+B u \\
& y=C x+D u
\end{aligned}
$$

Eşitlik 2'ye göre ÇETS sistem değişkenlerini uyarladığımızda sistemin giriş ve çıkış parametreleri Eşitlik 3'te verilmektedir. Eşitlik 3 'te verilen parametreler ÇETS sistemine göre uyarlanıp Eşitlik 2'ye göre düzenlendiğinde sistemin uzay-durum modeli Eşitlik 4 olarak bulunmaktadır.

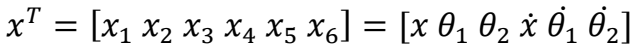

$$
\begin{aligned}
& y^{T}=\left[\begin{array}{lll}
x_{1} & x_{2} & x_{3}
\end{array}\right]=\left[\begin{array}{lll}
x & \theta_{1} & \theta_{2}
\end{array}\right]
\end{aligned}
$$

Eşitlik 4'te verilen A ve B matrislerindeki değerler sistemin dinamik modelinden elde edilmektedir ve Eşitlik 5'te verilmektedir.

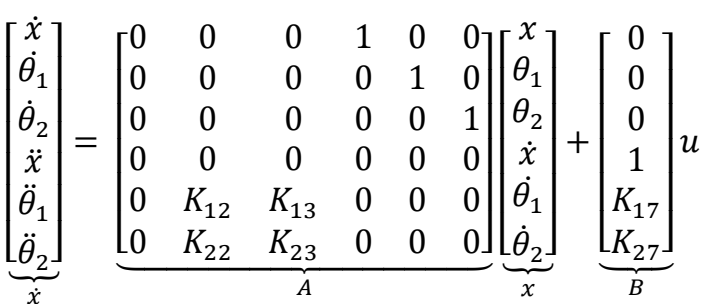




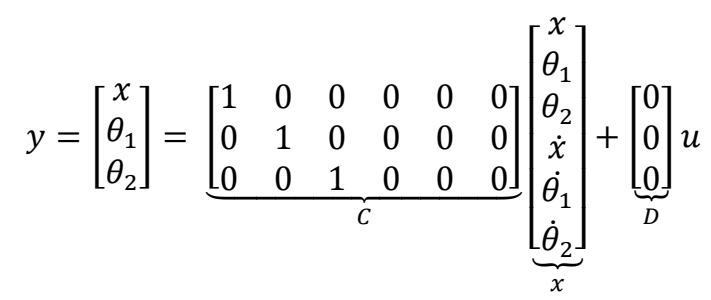

Çift eklemli ters sarkacın denge sürecinde genellikle $\cos \left(\theta_{1}-\theta_{2}\right)=1, \sin \left(\theta_{2}-\theta_{1}\right) \cong \theta_{2}-\theta_{1}, \cos \left(\theta_{2}\right) \cong \cos \left(\theta_{1}\right) \cong 1$, $\sin \left(\theta_{2}\right) \cong \theta_{2}, \sin \left(\theta_{1}\right) \cong \theta_{1}$ olduğundan tüm başlangıç değerleri " 0 " seçilmiştir.

$$
\begin{aligned}
& K_{12}=\left.\frac{\partial f_{1}}{\partial \theta_{1}}\right|_{x=0, \theta_{1}=0, \theta_{2}=0, \dot{x}=0, \dot{\theta}_{1}=0, \dot{\theta}_{2}=0, \ddot{x}=0}=\frac{3\left(-2 g m_{1}-4 g m_{2}-4 g m_{3}\right)}{2\left(-4 m_{1}-3 m_{2}-12 m_{3}\right) l_{1}} \\
& K_{13}=\left.\frac{\partial f_{1}}{\partial \theta_{2}}\right|_{x=0, \theta_{1}=0, \theta_{2}=0, \dot{x}=0, \dot{\theta}_{1}=0, \dot{\theta}_{2}=0, \ddot{x}=0}=\frac{9 g m_{2}}{2\left(-4 m_{1}-3 m_{2}-12 m_{3}\right) l_{1}} \\
& K_{17}=\left.\frac{\partial f_{1}}{\partial \ddot{x}}\right|_{x=0, \theta_{1}=0, \theta_{2}=0, \dot{x}=0, \dot{\theta}_{1}=0, \dot{\theta}_{2}=0, \ddot{x}=0}=\frac{3\left(-2 m_{1}-m_{2}-4 m_{3}\right)}{2\left(-4 m_{1}-3 m_{2}-12 m_{3}\right) l_{1}} \\
& K_{22}=\left.\frac{\partial f_{2}}{\partial \theta_{1}}\right|_{x=0, \theta_{1}=0, \theta_{2}=0, \dot{x}=0, \dot{\theta}_{1}=0, \dot{\theta}_{2}=0, \ddot{x}=0}=\frac{2 g\left(m_{1}+2\left(m_{2}+m_{3}\right)\right)}{4 m_{2} l_{2}-\frac{16}{9}\left(m_{1}+3\left(m_{2}+m_{3}\right)\right) l_{2}} \\
& K_{23}=\left.\frac{\partial f_{2}}{\partial \theta_{2}}\right|_{x=0, \theta_{1}=0, \theta_{2}=0, \dot{x}=0, \dot{\theta}_{1}=0, \dot{\theta}_{2}=0, \ddot{x}=0}=\frac{4 g\left(m_{1}+3\left(m_{2}+m_{3}\right)\right)}{3\left(4 m_{2} l_{2}-\frac{16}{9}\left(m_{1}+3\left(m_{2}+m_{3}\right)\right)\right) l_{2}} \\
& K_{27}=\left.\frac{\partial f_{2}}{\partial \ddot{x}}\right|_{x=0, \theta_{1}=0, \theta_{2}=0, \dot{x}=0, \dot{\theta}_{1}=0, \dot{\theta}_{2}=0, \ddot{x}=0}=\frac{2\left(m_{1}+2\left(m_{2}+m_{3}\right)\right)-\frac{4}{3}\left(m_{1}+3\left(m_{2}+m_{3}\right)\right)}{4 m_{2} l_{2}-\frac{16}{9}\left(m_{1}+3\left(m_{2}+m_{3}\right)\right) l_{2}}
\end{aligned}
$$

Buradan hareketle sistem uzay durum modeli Eşitlik 4 ve Eşitlik 5'e göre hesaplandığında sistemin uzay durum eşitliği yani $(\dot{x}=$ $A x+B u)$ Eşitlik 6 olarak bulunmaktadır.

$$
\left[\begin{array}{c}
\dot{x} \\
\dot{\theta}_{1} \\
\dot{\theta}_{2} \\
\ddot{x} \\
\ddot{\theta}_{1} \\
\ddot{\theta}_{2}
\end{array}\right]=\left[\begin{array}{cccccc}
0 & 0 & 0 & 1 & 0 & 0 \\
0 & 0 & 0 & 0 & 1 & 0 \\
0 & 0 & 0 & 0 & 0 & 1 \\
0 & 0 & 0 & 0 & 0 & 0 \\
0 & 77.0642 & -21.1927 & 0 & 0 & 0 \\
0 & -38.5321 & 37.8186 & 0 & 0 & 0
\end{array}\right]\left[\begin{array}{c}
x \\
\theta_{1} \\
\theta_{2} \\
\dot{x} \\
\dot{\theta}_{1} \\
\dot{\theta}_{2}
\end{array}\right]+\left[\begin{array}{c}
0 \\
0 \\
0 \\
1 \\
5.7012 \\
-0.0728
\end{array}\right] u
$$

Eşitlik 6'da elde edilen matrisler özdeğeri (Eigen) MATLAB üzerinden alındığında $0,0,0,0,-6.2074 \mathrm{i}$ ve $+6.2074 \mathrm{i}$ olarak bulunmaktadır. Bu işlem sonucunda sistemin kararsız yapıya sahip olduğu tespit edilmektedir. MATLAB üzerinden Eşitlik 7'de verilen sisteme ait kontrol matrisi (Controllable) $P_{c}$ ve gözlemci matrisi (Observable) $Q_{0}$ ile sistemin kontrol edilebilirliği test edilmiş̧tir. Sonuç olarak sistemin kontrol edilebileceği ancak kararlı hale getirilerek kontrol edilebilmesi için harici bir kontrol sistemine ihtiyaç duyduğu görülmektedir.

$$
\begin{aligned}
& P_{C}=\left[\begin{array}{llllll}
B & A B & A^{2} B & A^{3} B & A^{4} B & A^{5} B
\end{array}\right], \operatorname{rank}\left(P_{C}\right)=6 \\
& Q_{0}=\left[\begin{array}{lllll}
C & C A & \cdots & C A^{n-1}
\end{array}\right], \operatorname{rank}\left(Q_{0}\right)=6
\end{aligned}
$$

\section{PID ve LQR Tasarımlarının Gerçekleştirilmesi}

PID kontrolcüler endüstriyel uygulamalarda yoğun olarak kullanılan bir kontrol yöntemidir. Yapısının basitliği, diğer yöntemlere oranla daha az değiş̧ken içermesi ve parametre hesaplamalarındaki kolaylıkları sebebiyle günümüzde otomatik kontrol uygulamalarında en çok tercih edilen kontrol yöntemidir (Åström et al., 2006). PID kontrol yöntemi genel olarak doğrusal sistemlerde kullanılmasına rağmen birçok doğrusal olmayan sistem kontrolünde kullanılmış ve istenilen parametre değerlerine sistemi ulaştırmıştır (ÇEVEN et al., 2016; Chang et al., 2010). Eşitlik 8'de PID kontrolcüye ait matematiksel ifade verilmektedir.

$$
C_{P I D(s)}=K_{p}\left(k+\frac{k_{i}}{s}+k_{d} s\right)
$$

Eşitlik 8'de verilen $K_{p}$ oransal kazanç, $k_{i}$ integral kazanç ve $k_{d}$ türev kazanç sabiti olarak verilmektedir. Matlab/Simulink üzerinde modellenen ÇETS sisteminde PID kontrol yöntemi sistemin konum (x), alt sarkaç açısı $\left(\theta_{1}\right)$ ve üst sarkaç açısını $\left(\theta_{2}\right)$ "0" referans değerine başarıyla ulaştırmıştır. PID parametrelerinin hesaplanması Matlab/PID/Tuning araç kutusu yardımıyla hesaplanmıştır. Şekil 2'de sistemin PID ile kontrolüne ait Simulink modeli verilmektedir. Şekil 3'te PID kontrol için modellenen ÇETS sistemine ait Simulink blokları verilmektedir. Bloklar oluşturulurken Eşitlik 1 referans alınmıştır. 


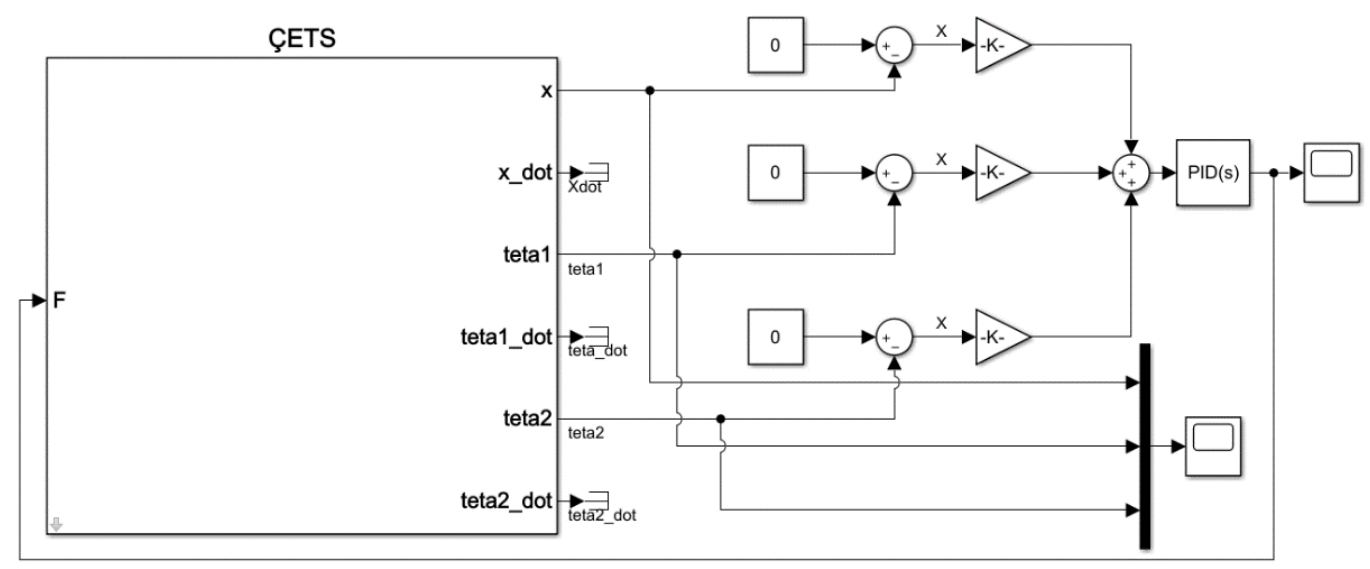

Şekil 2. ÇETS'ın PID ile kontrol modeli

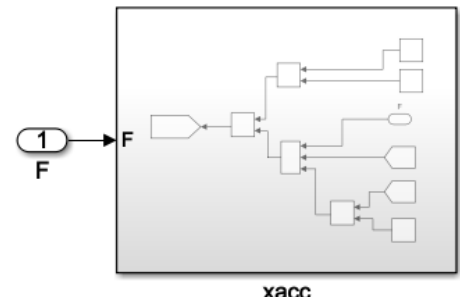

xacc

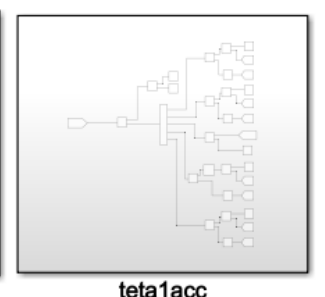

teta1acc

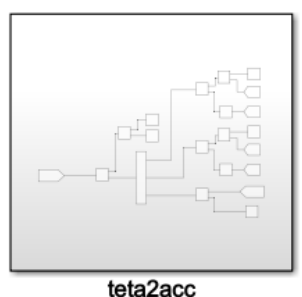

teta2acc

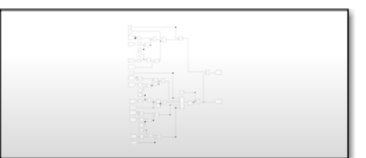

N1,N2

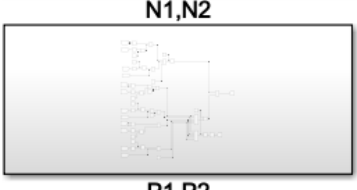

$\mathrm{P} 1, \mathrm{P} 2$

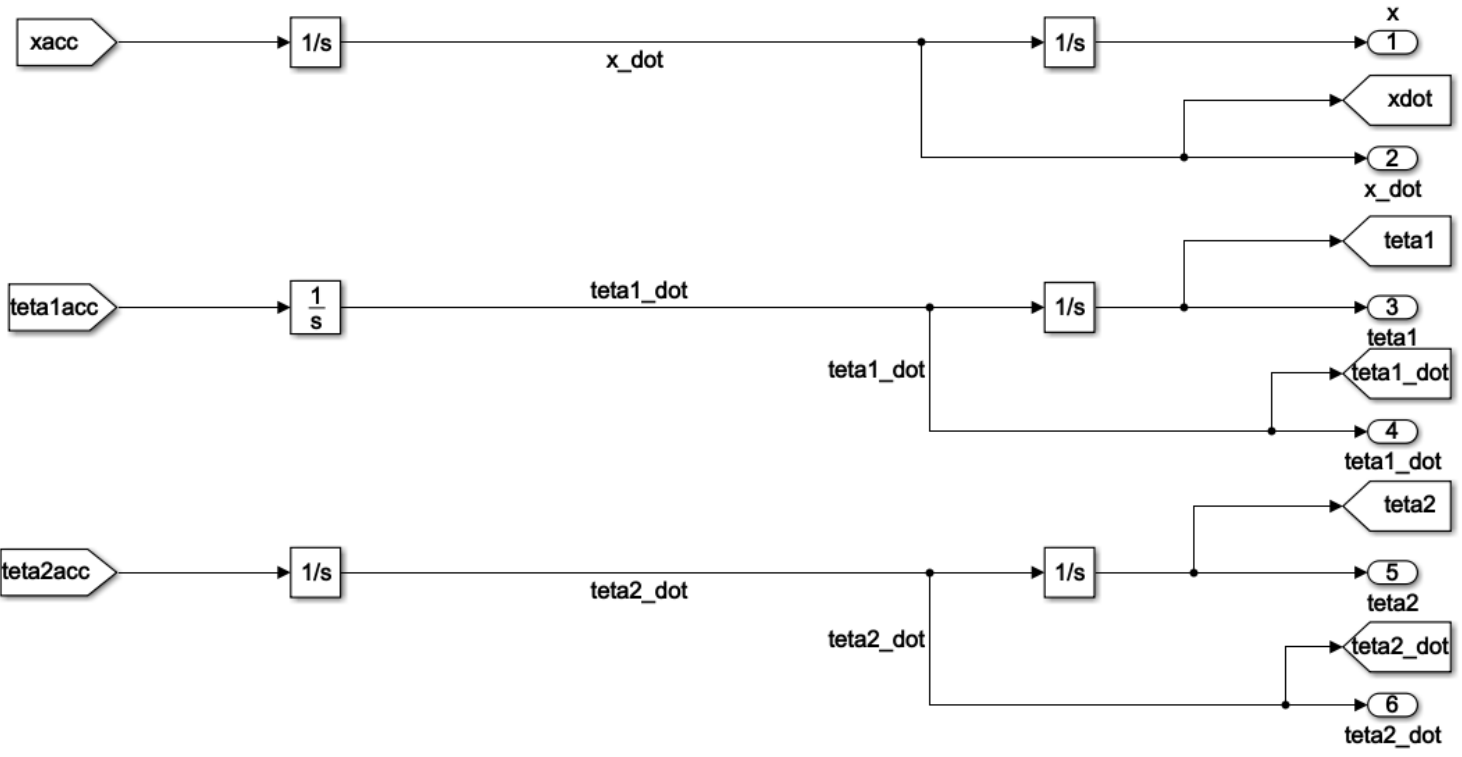

Şekil 3. ÇETS sistemi Simulink modeli

LQR kontrol yöntemi, belirlenen maliyet fonksiyonunu minimize edecek şekilde tasarlanan bir durum geri beslemeli en iyileme yapabilen kontrol yöntemidir (Purnawan et al., 2017). LQR kontrol yönteminde performans endeksinin $\left(U_{L Q R}\right)$ minimize edilmesi kontrolün temel amacıdır. LQR kontrolcüye ait performans denklemi Eşitlik 9'da verilmektedir.

$$
J_{L Q R}=\frac{1}{2} \int_{0}^{\infty}\left[x^{T} Q x+u^{T} R u\right] d t
$$

Eşitlik 9'da verilen $Q$ pozitif tanımlı matris ve $R$ pozitif yarı tanımlı matris $J_{L Q R}$ 'yi minimize edecek şekilde seçilmelidir. En iyilemeli kontrol için Eşitlik 10'da verilen P matrisi Riccati eşitliği ile sağlanmaktadır. Riccati eşitliği Eşitlik 11'da verilmektedir. Kontrolü için seçilen $Q$ ve $R$ matrisleri Eşitlik 12'de verilmektedir. Sonuç olarak LQR kontrolcünün $K$ kazanç matrisi Eşitlik 9 ile Matlab üzerinde hesaplanmaktadır. Kontrolcüye ait kazanç matrisi Eşitlik 13'te verilmektedir. Şekil 4'te LQR kontrol yapısı verilmektedir. 


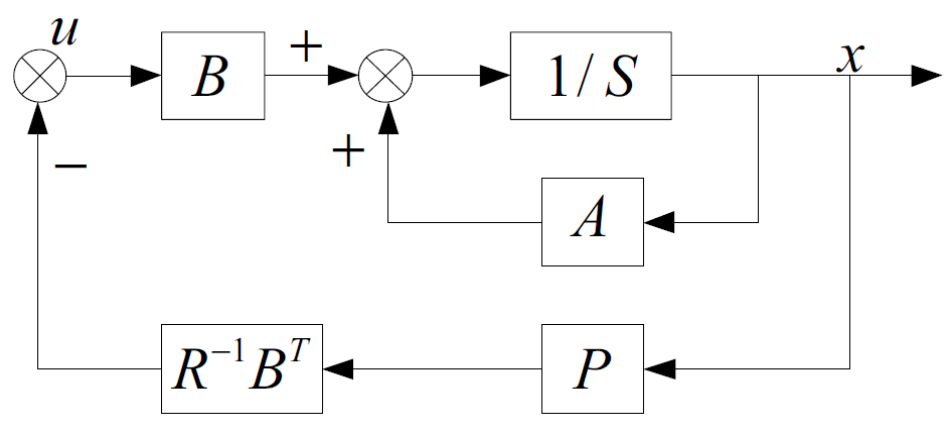

Şekil 4. LQR kontrol yapısı

$u^{*}(t)=-R^{-1} B^{T} P x(t)=-K x(t)$

$A^{T} P+P A-P B R^{-1} B^{T} P+Q=0$

$Q=\operatorname{diag}(7200,36,900,252,18,36), R=5.5$

$K=R^{-1} B^{T} P=[36.1814,132.2711,-274.7866,34.2128,0.7833,-47.0617]$

LQR kontrolcüye ait kazanç matrisi hesaplandıktan sonra LQR yöntemi için Simulink üzerinde uzay-durum modeli ile modellenen ÇETS sisteminde geri besleme fonksiyonu olarak kullanılmış ve yapılan deneysel çalışmalar sonucundan modelin başarıyla sistemi referans değere ulaştırdığı görülmüştür. Şekil 5’te LQR kontrol için Simulink üzerinde modellenen ÇETS ve LQR kazanç matrisinin geri besleme olarak kullanılmasına ait bloklar verilmektedir.

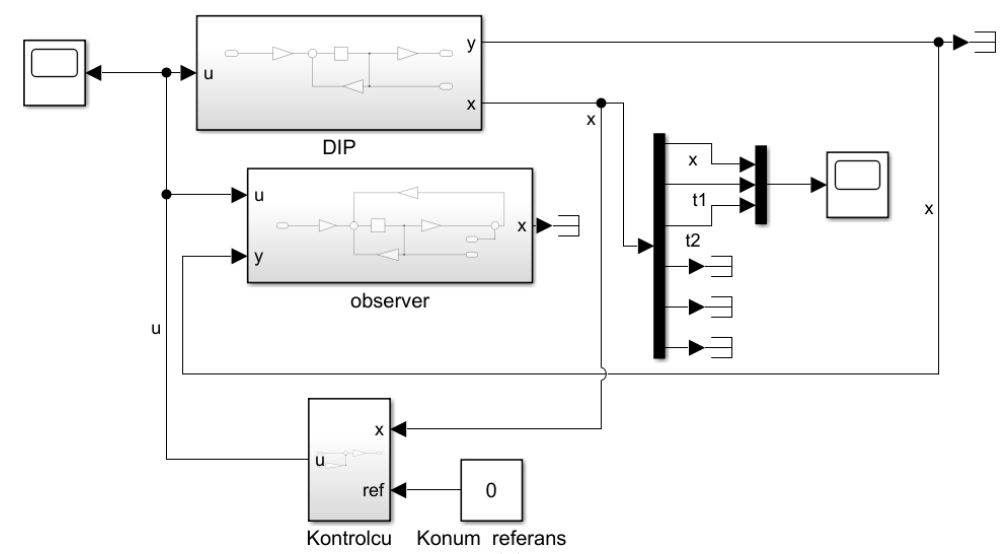

(a)

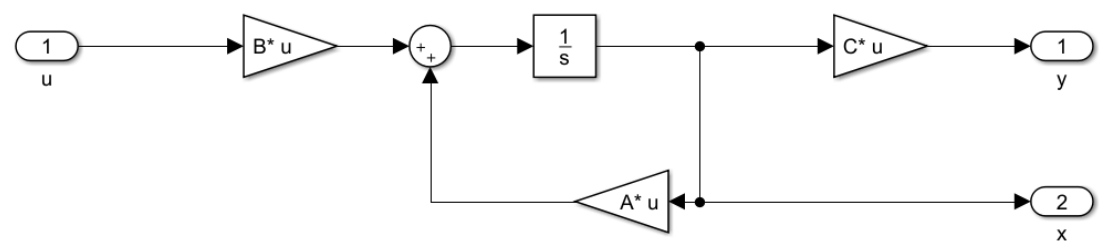

(b)

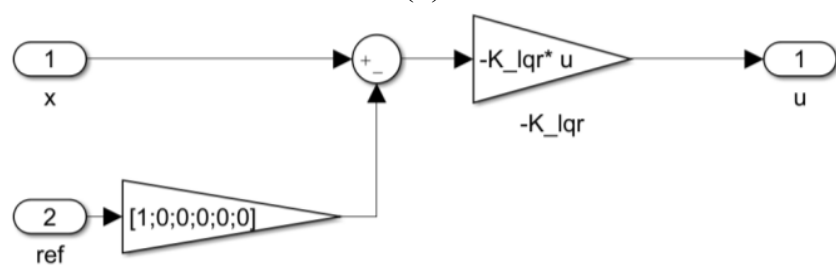

(c)

Şekil 5. ÇETS- LQR modeli, (a) Sistem modeli, (b) ÇETS uzay-durum modeli, (c) LQR kazanç modeli.

\section{Deneysel Çalışmalar}

ÇETS sistemi üzerinde uygulanması için tasarlanan PID ve LQR kontrol yöntemlerinin performanslarının izlenmesi ve karşılaştırılması amacıyla Matlab/Simulink üzerinde simülasyon çalışmaları yapıılmış ve sistemde konum (x), alt sarkaç açısı $\left(\theta_{1}\right)$ ve üst sarkaç açısı $\left(\theta_{2}\right)$ parametrelerine ait durumları izlenmiştir. Her iki kontrolcü için de sistem referans parametresi olarak konum seçilmiş ve istenilen konuma başlangıçta dengede olan sistemin ulaştırılması amaçlanmıştır. Simülasyonda sistemin başlangıç parametreleri $x=\left[x=0.3, \theta_{1}=0, \theta_{2}=0, \dot{x}=0, \dot{\theta}_{1}=0, \dot{\theta}_{2}=0\right]$ olarak seçilmiştir. Her iki kontrolcünün de sistemi dengeli olarak 
$x=0$ noktasına ulaştırması istenmektedir. Gerçekleştirilen deneyler sonucunda PID ve LQR kontrolcüleri referans konum noktasına başarıyla ÇETS'i dengeli bir şekilde ulaştırmıştır. Simülasyon süreci boyunca sistem konum parametresinin (x) zamana bağlı grafiği Şekil 6'da, alt sarkaç açısının $\left(\theta_{1}\right)$ zamana bağlı grafiği Şekil 7'de, üst sarkaç açısının $\left(\theta_{2}\right)$ zamana bağlı grafiği Şekil 8'de verilmektedir.

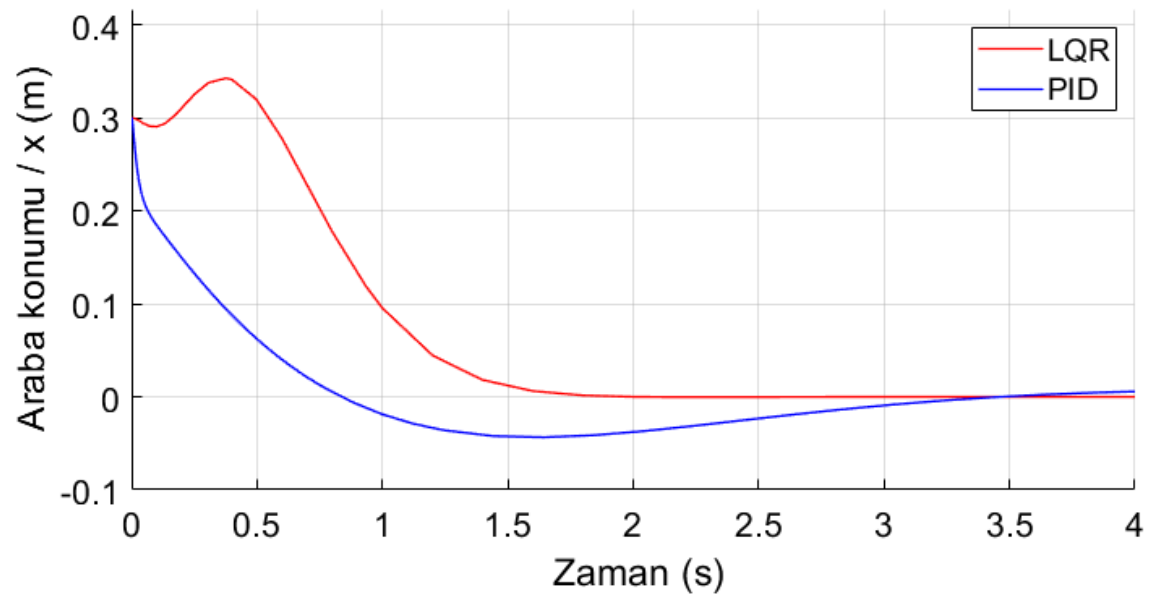

Şekil 6. ÇETS sistem konumu (x)

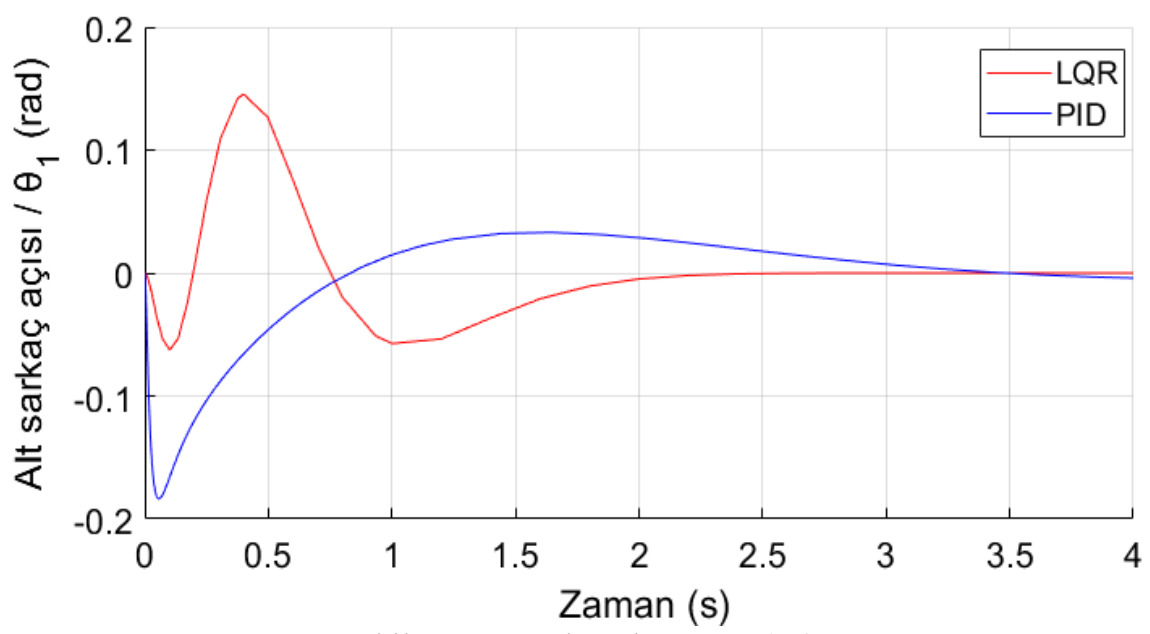

Şekil 7. ÇETS alt sarkaç açısı $\left(\theta_{1}\right)$

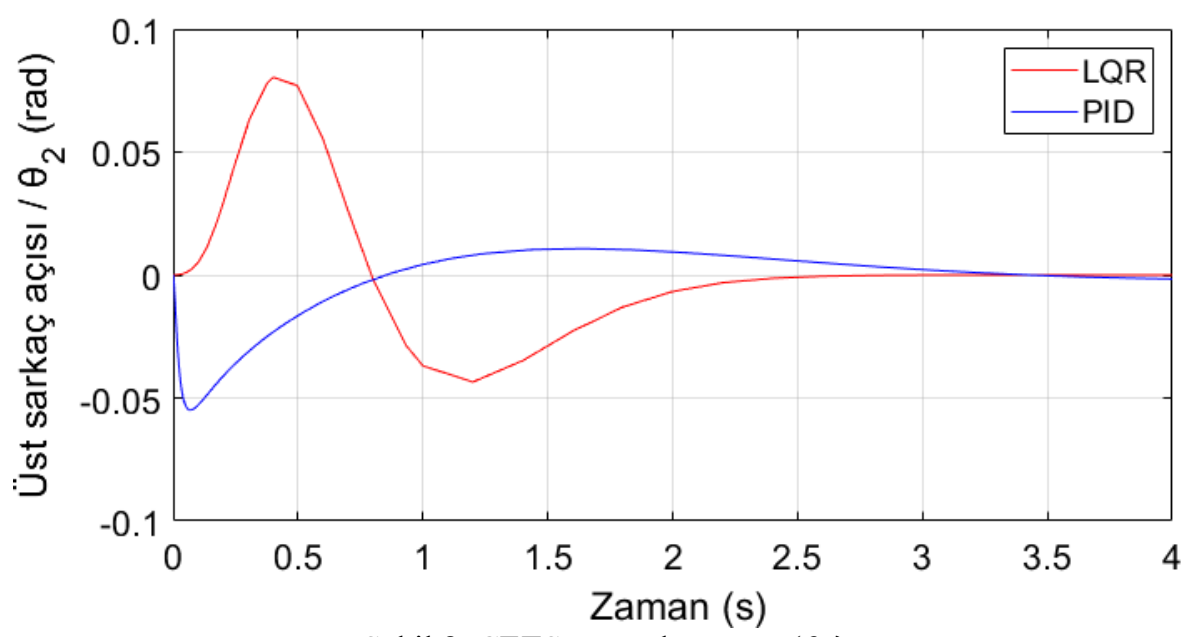

Şekil 8. ÇETS üst sarkaç açısı $\left(\theta_{2}\right)$

\section{Sonuçlar}

Otomatik kontrol yöntemleri günümüzde birçok sistemin insansız kontrolünün sağlanmasında kullanılmaktadır. Özellikle gelişen teknoloji ve insan faktörünün sistemde en aza indirgenmeye çalışıldığı günümüzde, sistemlerin karmaşıklaşması nedeniyle var olan kontrol yöntemlerinin geliştirilmesi ve bu yolla daha başarılı sonuçlar elde edilmesi amaçlanmaktadır. Ters sarkaç sistemleri yapılarının karmaşıklığı ve kontrollerinin zorluğu sebebiyle birçok kontrol yöntemi geliştirme çalışmalarında yoğun olarak kullanılmaktadır. 
Bu çalışmada, PID ve LQR kontrol yöntemleri kararsız ve doğrusal olmayan bir sistem üzerinde uygulanmış, sonuçlar incelenmiş ve yöntemlerin kıyaslaması yapılmıştır. Yöntemlerin kıyaslanması bir ÇETS modeli üzerinde yapılmıştır. Çalışmalardan elde edilen sonuçlara göre, iki yöntem de başlangıçta dengede bulunan sarkacı hedef noktaya dengeli halde ulaştırmıştır. PID yöntemi ile yapılan çalışmalarda sistemde alt sarkaç ve üst sarkaç açılarında salınım, LQR yöntemi ile yapılan çalışmalardaki salınımdan az olmasına rağmen referans değer parametrelerine oturma zamanının yüksek olduğu görülmüştür. Ayrıca dinamik bozucu etkilerde sistemin tepki verme hızı ve referans değere ulaşması LQR yöntemine göre daha düşük olacağı için kontrolün zor olabileceği düşünülmektedir. LQR yöntemi ile gerçekleştirilen simülasyonlarda sistem hedef konuma $\mathrm{t}=2 \mathrm{~s}$ de ulaşırken, PID yönteminde $\mathrm{t}=4 \mathrm{~s}$ olarak simüle edilmektedir. Ayrıca alt sarkaç ve üst sarkaç açıları referans değerlere LQR yönteminde $t=2.5 \mathrm{~s}$ de ulaşırken, PID yönteminde $\mathrm{t}=4 \mathrm{~s}$ olarak simüle edilmektedir. Sonuç olarak ÇETS sistemlerinde konum ve denge kontrolü için daha hızlı tepki vermesi nedeniyle LQR yönteminin kullanılması önerilmektedir.

\section{Kaynakça}

Arbo, M. H., Raijmakers, P. A., \& Mladenov, V. M. (2014). Applications of neural networks for control of a double inverted pendulum. 12th Symposium on Neural Network Applications in Electrical Engineering (NEUREL), 89-92.

Åström, K. J., Hägglund, T., \& Astrom, K. J. (2006). Advanced PID control (Vol. 461). ISA-The Instrumentation, Systems, and Automation Society Research Triangle $\square$.

Çeven, S., \& Bayır, R. (2016). Implementation of Fuzzy Logic Based Speed Control of Brushless Direct Current Motors via Industrial PC. International Journal of Intelligent Systems and Applications in Engineering, 146-152.

Chang, W.-D., \& Shih, S.-P. (2010). PID controller design of nonlinear systems using an improved particle swarm optimization approach. Communications in Nonlinear Science and Numerical Simulation, 15(11), 3632-3639.

Ding, C. J., Ping, D., Zhang, M. L., \& Zhang, Y. F. (2009). Double inverted pendulum system control strategy based on fuzzy genetic algorithm. Proceedings of the 2009 IEEE International Conference on Automation and Logistics, ICAL 2009, $2007,1318-1323$. doi: 10.1109/ICAL.2009.5262779

Nejadfard, A., Yazdanpanah, M. J., \& Hassanzadeh, I. (2013). Friction compensation of double inverted pendulum on a cart using locally linear neuro-fuzzy model. Neural Computing and Applications, 22(2), 337-347.

Önen, Ü., Çakan, A., \& İLhan, İ. (2019). Performance comparison of optimization algorithms in LQR controller design for a nonlinear system. Turkish Journal of Electrical Engineering and Computer Sciences, 27(3), 1938-1953. doi: 10.3906/elk-1808-51

Priyadarshi, P. (2013). Optimal Controller Design for Inverted Pendulum System: An Experimental Study.

Purnawan, H., Purwanto, E. B., \& others. (2017). Design of linear quadratic regulator (LQR) control system for flight stability of LSU05. Journal of Physics: Conference Series, 890(1), 12056.

Sheng, Q., Qing, Z., Gao, X. Z., \& Shuanghe, Y. (2008). ANFIS controller for double inverted pendulum. IEEE International Conference on Industrial Informatics (INDIN), 475-480. doi: 10.1109/INDIN.2008.4618147

Sun, Z., Wang, N., \& Bi, Y. (2015). Type-1/type-2 fuzzy logic systems optimization with RNA genetic algorithm for double inverted pendulum. Applied Mathematical Modelling, 39(1), 70-85.

Tinkir, M., Kalyoncu, M., Onen, U., \& Botsali, F. M. (2010). PID and interval type-2 fuzzy logic control of double inverted pendulum system. 2010 The 2nd International Conference on Computer and Automation Engineering (ICCAE), 1, 117-121.

Wu, J., Liu, C., \& Deng, Y. (2008). Variable Structure Control for Stabilizing Double Inverted Pendulum. 2008 International Conference on Intelligent Computation Technology and Automation (ICICTA), 1, 741-744.

Zhang, S., Zhang, Z., Jin, K., \& Yang, C. (2012). Fuzzy control of double inverted pendulum by using state varieties fusion function. Proceedings of the 2012 24th Chinese Control and Decision Conference, CCDC 2012, 2696-2699. doi: 10.1109/CCDC.2012.6244428

Zheng, Y., Zhong, P., \& Yue, Q. (2016). Double Inverted Pendulum Based on LQG Optimal Control. Icacie, 83-87. doi: 10.2991/icacie16.2016.20 